\title{
Waste Classification using Deep Learning Convolution Neural Nets
}

Divy Mohan Rai ${ }^{1}$ | Ms. ShikhaGupta 2

${ }^{1}$ B. Tech Scholar, Department of IT, Maharaja Agrasen Institute of Technology, Delhi, India

${ }^{2}$ Assistant Professor, Department of IT, Maharaja Agrasen Institute of Technology, Delhi, India

\section{To Cite this Article}

Divy Mohan Rai and Ms. ShikhaGupta, "Waste Classification using Deep Learning Convolution Neural Nets", International Journal for Modern Trends in Science and Technology, 6(12): 449-454, 2020.

\section{Article Info}

Received on 16-November-2020, Revised on 09-December-2020, Accepted on 12-December-2020, Published on 19-December-2020.

\section{ABSTRACT}

The generation of waste India is becoming a great concern, and it has affected our environment and may even affect the life of people living near these dump sites. The recent study figures show that India generates nearly 26,000 MT of plastic waste on a daily basis and 94 lakh tonnes trash every year. In 2017-18, the Capital generated 6,800 tonnes of municipal waste daily, out of which 690 tonnes of trash was plasticthe highest in the country. This project focuses on building a Deep learning classifier to classify a given waste product into one of the recycling categories such as plastic, glass so that it is easier for people to recycle waste and less waste reaches the wrong place like illegal dumpsites which can lead to not only health issues but also cause a big damage to our surrounding environment. The model is based on the InceptionV3 architecture and uses the concept of transfer learning for training. The system has been tested on the dataset collected from various resources ranging from the TACO dataset to the TrashNet dataset the overall accuracy of the model was around $94.6 \%$.

KEYWORDS: Convolutional Neural Networks, Pre-train Model, Waste Separation, Automation, Machine Learning

\section{INTRODUCTION}

The world bank report showed that there are almost 4 billion tons of waste around the world every year and the urban alone contributes a lot to this number, the waste is predicted to increase by 70 percent in the year 2025. According to world bank in the next 25 years, the less developed countries' waste accumulation will increase drastically. With the increase in the number of industries

intheurbanarea, thedisposalofthesolidwasteisreally becomingabigproblem, andthesolidwasteincludesp aper,wood,plastic, metal, glass etc. The main method of managing the waste is landfilling, which is inefficient and expensive and polluting natural environment. For example, the landfill site can affect the health of the people who stay around the landfill site. Another common way of managing waste is burning waste and this method can cause air pollution and some hazardous materials from the waste spread into the air which can cause cancer. Hence it is necessary to recycle the waste to protect the environment and human beings' health, and we need to separate the waste into the different components which can be recycled using differentways. 


\subsection{Motivation}

The presentway of separating waste/garbage is the hand-picking method, whereby someone is employed to separateout the differentobjects/materials. Theperson, whoseparate waste, ispronetodiseasesduetotheharmfulsubstanc esinthegarbage.With

thisinmind,itmotivatedustodevelopanautomatedsy stemwhichisabletosortthewaste.andthissystemcan takeshorttimeto

sortthewaste, anditwillbemoreaccurateinsortingtha nthemanualway. Withthesysteminplace, thebenefici alseparatedwaste can still be recycled and converted to energy and fuel for the growth of the economy.

This product will not only help the user to dispose of an already bought product, the user can use it at the time of his/her purchase to determine whether an item is recyclable or not and reduce the amount of non-recyclable product that they buy. To explain let's imagine a scenario where the user goes to a shopping mart to buy a product, he/she is not very aware of the symbols mentioned at the bottom of the product they use the classifier to determine the nature of the product in an easy way that helps them with their purchase.

\subsection{Related Works}

Many different algorithms have been developed for the classification of images, such has RNNs, SVMs, ANN etc, but Convolutional Neural Network which is a Machine Learning algorithm has really performed better than them all. CNNs hit the spot when the algorithm was used to win the 2012 image-Net large-scale visual recognition challenge(ILSVRC) which was proposed in. Since 2012 many different CNN architectures have been developed which has solved many image classification

problems.LuleaUniversityoftechnologyin 1999unde rtookaproject, andasystemwasdevelopedtorecyclem etal scraps using mechanical shape identifier.The model will be made using the InceptionV3 architecture and based on the concept of transfer learning. Inception-v3 is a convolutional neural network that is 48 layers deep

\subsection{Dataset}

For this work, we are using a trash image dataset. We also use the TACO dataset that is available online. This is a small data, which is divided into mainly four different classes glass, paper, plastic, metal, all the pictures of the images have been resized down to $512 \times 384$.

\section{Methodology}

II.

For the pre-processing stage, data augmentation method was performed on the images, because of the small size. This technique was chosen because of the different orientations of the waste materials. Some of the technique includes, random of the image,

translatingtheimage, randomlyscalingtheimage, ima geshearing,randomlyscalingoftheimage. Withthiste chniqueitmaximizes the dataset size. And helps in depicting the various scenarios where the user might click the picture.

\subsection{InceptionNetV3}

The model will be made using the InceptionV3 architecture and based on the concept of transfer learning. Inception-v3 is a convolutional neural network that is 48 layers deep. You can load a pretrained version of the network trained on more than a million images from the ImageNet database. The pretrained network can classify images into 1000 object categories, such as keyboard, mouse, pencil, and many animals. As a result, the network has learned rich feature representations for a wide range of images. The network has an image input size of 299-by-299.

\subsection{Experimentation}

The first stage for training the model using transfer learning is to freeze the initial layers and train some of the final layers. As the original inception model was trained on the ImageNet dataset it has 1000 nodes at the output layer. As here we are working with only 20 classes, we replace the last SoftMax layer with a SoftMax unit with 20 nodes only.

The initial model achieved an overall accuracy of around $86 \%$. The next stage was to unfreeze some more layers and retrain the model. Following this the model's accuracy was increased to $90 \%$ but the model started to overfit. 
To overcome this a dropout layer was added with a dropout probability of 0.3 which means out of every 10 nodes 3 will be dropped this helps the model to generalize rather than overfit on the training data. The accuracy was increased to $94 \%$ and further changes and iterations to the model did not have a significant effect on the accuracy.

Some of the images from the original dataset can be seen in the figure below along with their categories:
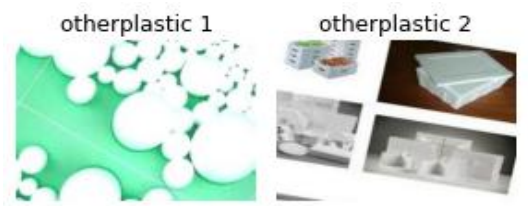

otherplastic 3
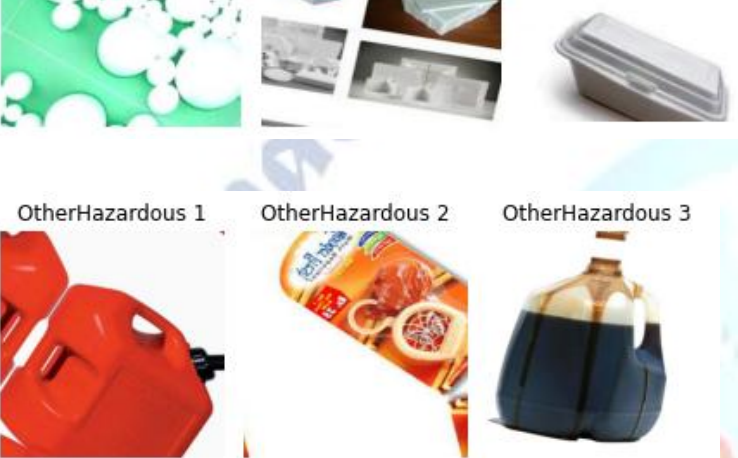

OtherHazardous 3
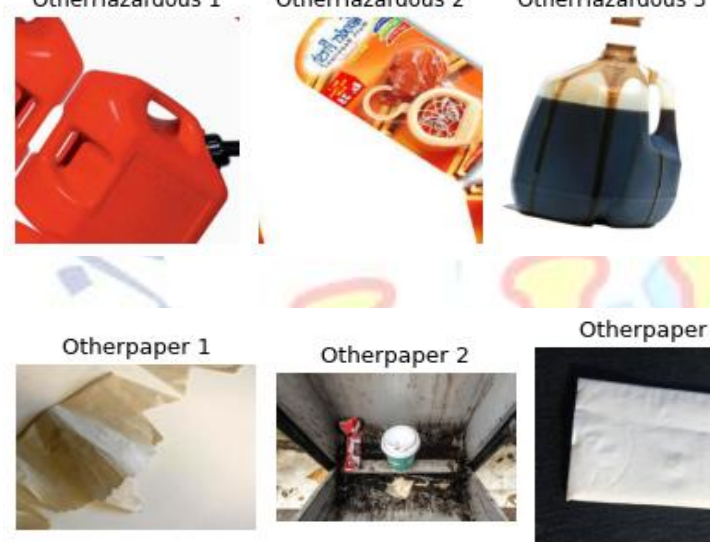

Otherpaper 3

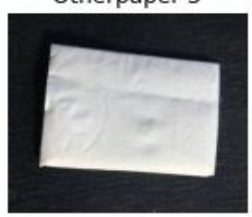

Battery 1
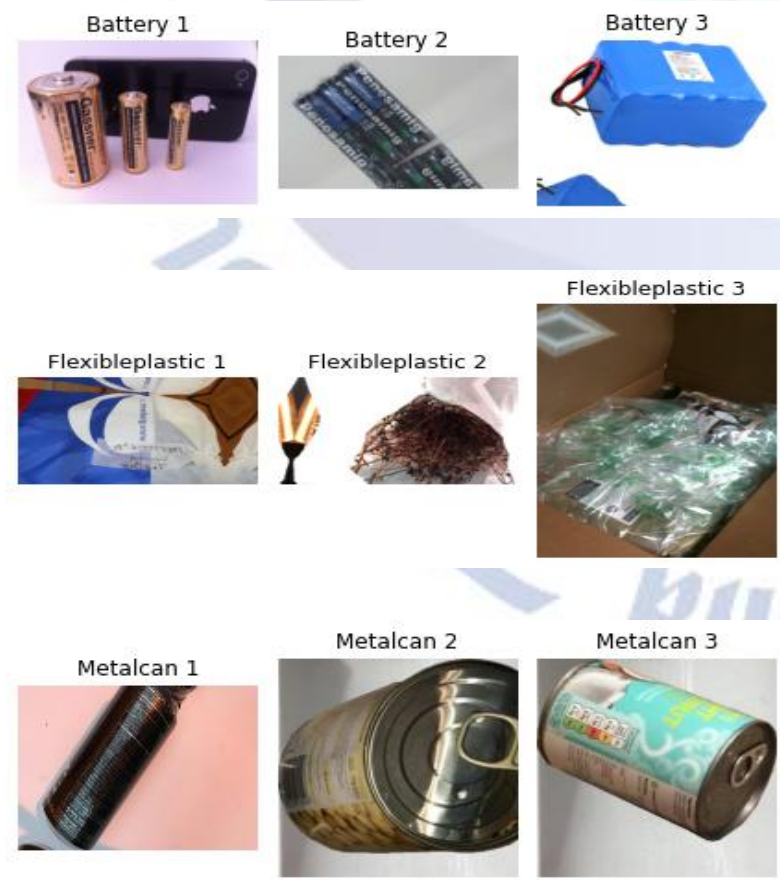
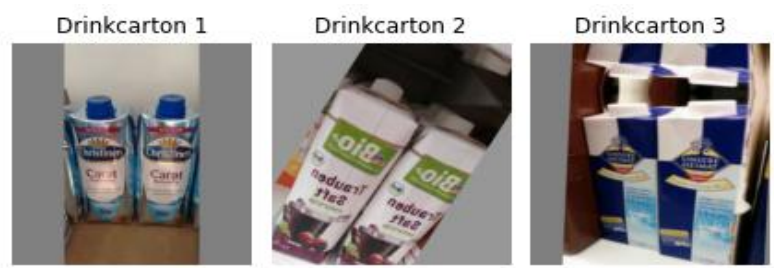

\section{1) Results}

The model achieved an overall accuracy of $94.6 \%$ in training phase on the testing data. The model was trained for around 3000 iterations in the test set

Here is a graph representing the overall training phase percentages:

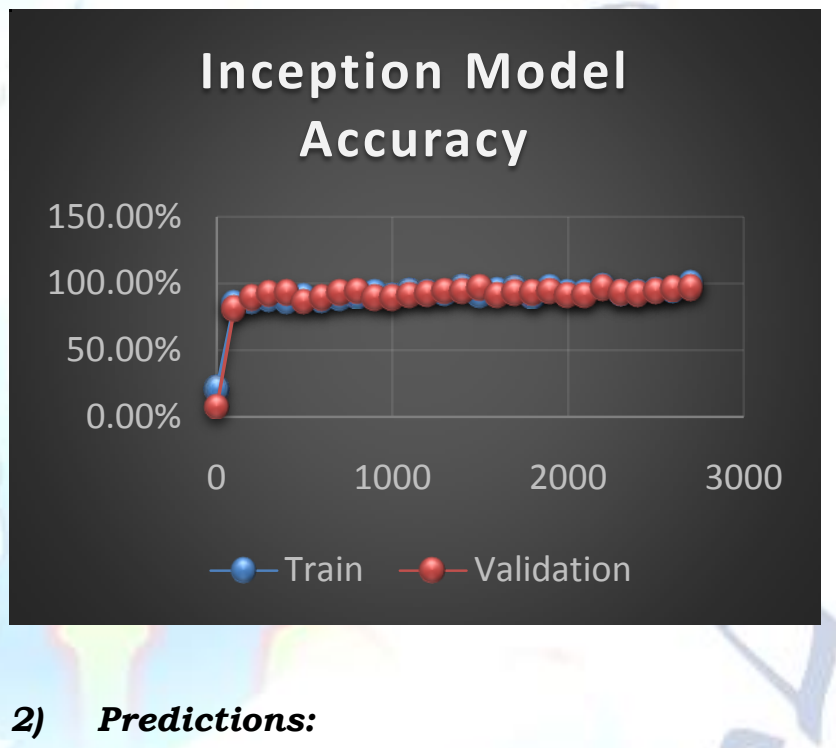

Here are some of the Sample predictions from the model:

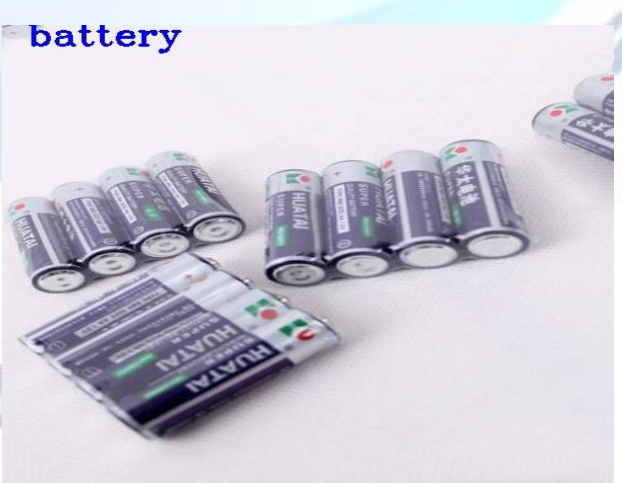



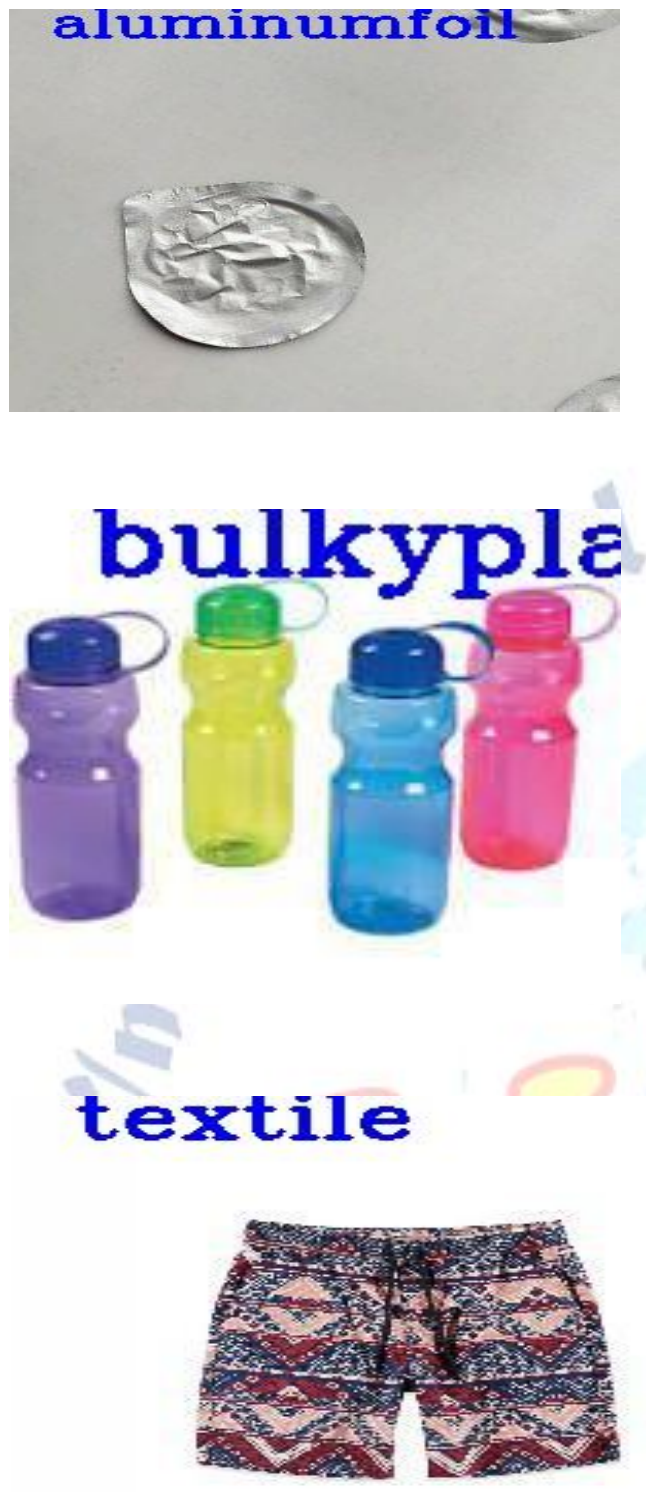

\section{Conclusion and Future Scopes:}

- Using data augmentation to increase the amount of training data from a few thousands to millions.

- Building a platform where the user will be presented with the choice of uploading the images and then he will also be given the proper guidelines for complete recycling of the product.

\section{References:}

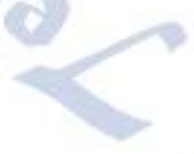

[1] D. Hoornweg and P. Bhada-Tata, "A Global Review of Solid Waste Management," (2012) 1-116. [2] Niehs.nih.gov, "Cancer and the Environment," 46, 2018. [Online]. Available: https://www.niehs.nih.gov/health/materials/can cer_and_the_environment_508.pdf.

[3] N. Johansson and H. Corvellec, "Waste policies gone soft: An analysis of European and Swedish waste prevention plans," Waste Manag., vol. 77, pp. 322-332, 77 (2018) 322-332.

[4] Y. Bengio, "Deep Learning," Www, 2017, pp. $1-66$.

[5] O. Kuchaiev and B. Ginsburg, "Factorization tricks for LSTM networks," 2016 IEEE Conf. Comput. Vis. Pattern Recognit., 2017, pp. 1-6. [6] https://opensource.google/projects/open-imagesdataset

[7] http:/ / tacodataset.org/

[8] https://github.com/garythung/trashnet [9[

https://www.indiatoday.in/india/story/india-s-tr ash-bomb-80-of-1-5-lakh-metric-tonne-daily-garb age-remains-exposed-untreated-1571769-2019-0 $7-21$
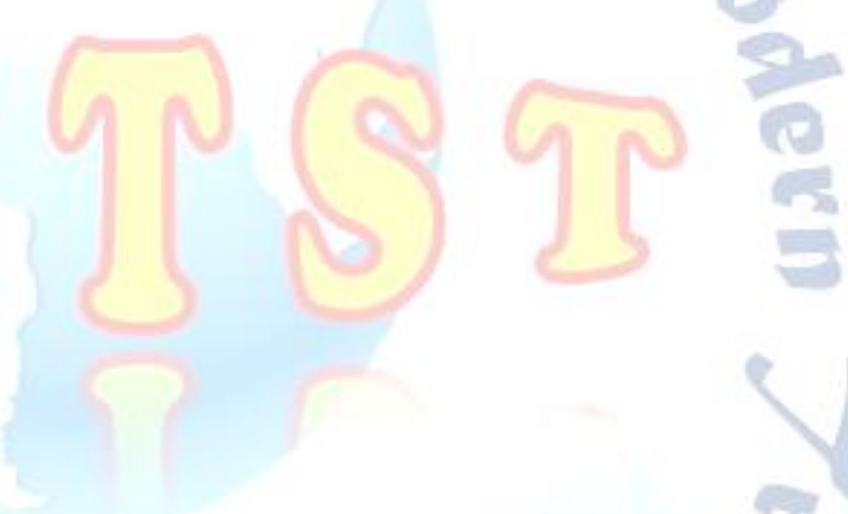